\title{
An Efficient DWT-DAPM Technique for PAPR Reduction in OFDM System
}

\author{
Puneeth Kumar D. N., M. N. Eshwarappa
}

\begin{abstract}
In this paper, Discrete Wavelet Transform (DWT) Orthogonal Frequency Division Multiplexing (OFDM) system is compared with Discrete Cosine Transform (DCT) and Discrete Fourier Transform (DFT) OFDM systems. The channel noise is modelled with A white Gaussian Model (AWGN), the fading is the impairment in the channel and modelled by Rayleigh fading which is frequency selective fading channel and flat fading channel. The comparisons of Peak to Average Power Ratio (PAPR) and Bit Error Rate (BER) are made using modulation techniques such as Differential Amplitude and Phase Modulation (DAPM), Quadrature Amplitude Modulation (QAM) and Pulse Amplitude Modulation (PAM). Simulation results shows that PAPR is $4.497 \mathrm{~dB}$ for DWT-DAPM combination, $4.684 \mathrm{~dB}$ for DWT-QAM combination and $6.211 \mathrm{~dB}$ for DWT- PAM combination at $10^{-3}$ Complementary Cumulative Distributive Function (CCDF).The performance Analysis with the combination of DFT, DCT with DAPM, QAM and PAM are also compared. The BER is $0.01816,0.01806$ at $20 \mathrm{~dB} S N R$ in frequency selective channel, flat fading channel for DWT-DAPM and for DWT- QAM, AWGN channel BER is 0.01765 at 20dB SNR.
\end{abstract}

Keywords: Orthogonal Frequency Division Multiplexing (OFDM), Discrete Wavelet Transform (DWT), Differential Amplitude and Phase Modulation (DAPM), Flat Fading Channel, Frequency Selective Fading Channel.

\section{INTRODUCTION}

In this communication era, OFDM is a modulation technique which is multi-carrier in nature which provides a powerful means to carry high speed data streams on a multipath fading, the path that causes Intersymbol interference (ISI) [1] . To remove ISI a cyclic prefix is added but, this can greatly reduce efficiency of bandwidth. In recent studies, DWT-OFDM system becomes substitute for the systems like DFT-OFDM and DCT-OFDM. In DWT-OFDM, the cyclic prefix is not required due to the overlapping techniques and provides robustness against ISI due to which the subcarriers are having finite length. The OFDM signal is composed by large peak amplitudes which results in increased amount of inter carrier modulation caused by the linear power amplifiers (LPA)[2], this results in large PAPR and BER. In

\section{Revised Manuscript Received on February 05, 2020.}

* Correspondence Author

Puneeth Kumar D. N.*, Research Scholar, Visveswaraya Technological University, Belagavi, India. Email: puneethdnkumar@gmail.com

M. N. Eshwarappa, Professor, Department of ECE, SSIT, Tumkur, India. Email: jenuece2016@gmail.com

(C) The Authors. Published by Blue Eyes Intelligence Engineering and Sciences Publication (BEIESP). This is an open access article under the CC BY-NC-ND license (http://creativecommons.org/licenses/by-nc-nd/4.0/) reference 3 , the DWT based OFDM provides a substitute for FFT based system in which PAPR is reduced by $2.3 \mathrm{~dB}$ over the standard FFT based system at $10^{-4}$ CCDF without decreasing the bandwidth efficiency [3]. In reference 4, by using DWT, BER is calculated which is of $10^{-3}$ at $12 \mathrm{~dB}$ of SNR by using QAM modulation technique [4]. In reference 5 , PAPR is between by $3.5-3.7 \mathrm{~dB}$ at $10^{-1}$ CCDF by using Haar, symlet, coiflet, dB2, Bio and Rbio wavelets. BER is also calculated in which Rbio wavelet has less BER than other wavelets [5]. In Reference 6, at $M=4$ PAPR is $7.4 \mathrm{~dB}$, where $\mathrm{M}$ is the number of carriers and it is done for different subcarriers [6]. In reference 7, SLM technique is used for Discrete Sine Transform (DST), DCT and DWT for PAPR reduction which is of $7.5 \mathrm{~dB}$ at CCDF of $10^{-3}$ [7]. In reference 8 , by using the pilot symbols, the OFDM symbols phase is rotated and this technique has an advantage that it has no added complexity and also for better PAPR reduction Symlet and Daubechies wavelets are used [8]. In reference 9, fourier transform and wavelet transform analysis are performed and the percentage changes of the values are obtained. The analysis is performed for SLM and PTS methods [9]. In reference 10 , the effect of different wavelet families such as Haar, Daubechies, Symlets, coiflets and Biorthogonal are analysed, the difference between the better PAPR value and less PAPR value is approximately about $4.5 \mathrm{~dB}$ for bior 2.4 and coif 1 wavelets [10]. From this literature, Wavelet is a promising technique that will reduce the PAPR better than the other techniques but still there are avenues that PAPR can be reduced by using the combination of different modulation techniques with wavelets.

In this paper, DWT- DAPM based OFDM system is proposed, in which 64 subcarriers and 1200 OFDM symbols are used. The performance evaluation for PAPR and BER is done for the proposed system with conventional methods such as DFT, DCT and modulation techniques like QAM and PAM in the AWGN channel, frequency selective fading and flat fading channel.

\section{PRINCIPLE CONCEPT OF OFDM SYSTEM}

DFT/FFT based OFDM system is shown in figure 1.Consider an input binary sequence and this sequence is serial and converted to $\mathrm{N}$ parallel data streams by demutiplexing. Each of these data streams is converted to symbol streams, after which IDFT/IFFT is performed for this set of samples to obtain complex set of time domain samples of the signal. These samples are converted into analog domain by using digital to analog converters. The transmission signal is obtained by summing of all the analog signals [3] 


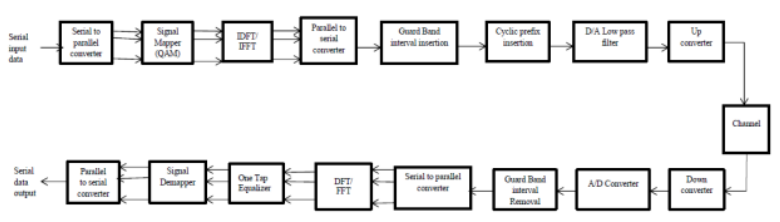

Fig. 1 Block diagram of FFT/DFT based OFDM System [3].

The duration of OFDM symbol is Tb seconds. The inverse Fourier transform signal in the time domain and with the time interval is represented as $\mathrm{Tb}=\mathrm{NT}$ and the spectrum is also represented as

$T_{b}=\frac{2 \pi}{\Delta \omega} \Leftrightarrow \Delta \omega=\frac{2 \pi}{T_{b}}=2 \pi \Delta f$

By using the equation (1), the Fourier series spectrum for the $\mathrm{j}^{\text {th }}$ OFDM symbol is given by

$U_{j}(\omega)=\sum_{p=0}^{N-1} U_{j}[p] \delta_{c}(w-p \Delta \omega)$

Equation (2) can be represented in time domain by

$b(t)=1 / \sqrt{T_{b}} \sum_{p=0}^{N-1} B_{p} e^{j 2 \pi f_{p} t} \quad 0 \leq \mathrm{t} \leq \mathrm{T}_{\mathrm{b}}$

Cyclic prefix is used in OFDM system to make channel estimation easy by combating multipath, for example consider an OFDM system which has $M$ subcarriers [3]. The message symbol can be written as $d=\left[\mathrm{d}_{0}, \mathrm{~d}_{1}, \ldots \ldots \ldots, \mathrm{d}_{\mathrm{M}-1}\right]^{\mathrm{T}}$ .The OFDM signal is obtained by taking the IDFT or IFFT of the message symbol followed by a cyclic prefixing which is shown in figure 2.

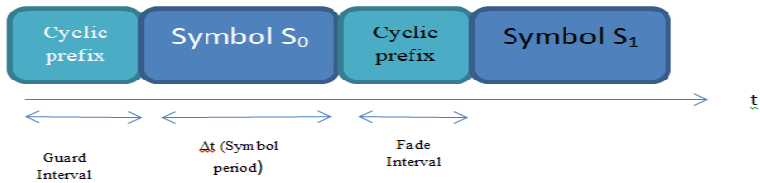

Fig. 2 Cyclic prefix of DFT/FFT based OFDM system [3]. Since OFDM symbols are long, ISI gets added, in order to remove ISI guard interval is added to the OFDM system [3]. In the transmitter of OFDM signal the power amplifier is operated near the saturation region for the efficiency of power and the varying signal amplitudes are sensitive due to the nonlinear characteristics. The variations in these signal amplitude causes at the power amplifier will cause ISI and high PAPR. The ISI increases BER and degrades the system performance $[11,12]$. Large PAPR requires large dynamic range analog to digital converters which are costly. Without compromising the BER, the system performance can be improved by reducing PAPR.

The PAPR is defined for the OFDM signal as,

$$
P A P R=10 \log _{10} \frac{\max \left(\left(b(t)^{2}\right)\right.}{E\left(\left(b(t)^{2}\right)\right.} \text { for } 0 \leq t \leq M T
$$

Where $\mathrm{T}$ is the symbol duration and $\mathrm{E}$ ( ) is the mathematical expectation
The CCDF is a performance measuring index which indicates the probability of PAPR exceeding a specified threshold. For $M$ subcarriers, the PAPR values depend on the nature of the wavelet basis used. Hence by properly selecting the wavelet basis, PAPR can be reduced. The CCDF is given by [15]

Probability $(\mathrm{PAPR}>\mathrm{Z})=1-\mathrm{P}(\mathrm{PAPR} \leq \mathrm{Z})$

Where $\mathrm{Z}$ is the PAPR threshold

\section{PROPOSED SYSTEM}

Wavelet is nothing but a small wave which is having finite duration. Wavelet is the basis for DWT. Unlike the sinusoidal signals which form the basis for the Fourier Transform, the wavelets are asymmetric, irregular and having finite duration. Due to the variations in time-frequency resolutions, the basis functions in terms of scale and frequency is a major advantage over Fourier transform. The wavelet basis function will divide the data into different frequency components and the components are chosen related to its scale. The wavelet basis function which is divided into different windows of different frequencies with variable size, causes the variations in time-frequency resolution and the Fourier basis function is divided into windows of square size which does not vary in time frequency resolution. Infinite number of basis function is provided with variation in time- frequency resolution but there is only one variation in Fourier Transform.

The mathematical representation of DWT and IDWT are shown in equations (6) and (7) respectively

$$
\begin{aligned}
& D_{k}=\sum_{k=0}^{N-1} d(k) \Psi(2 k-n) \\
& d(k)=\sum_{m=0}^{\infty} \sum_{n=0}^{\infty} D_{k} \Psi(2 k-n)
\end{aligned}
$$

Where $\Psi$ is the wavelet kernel and is selected according to the mother wavelet chosen.

The Block diagram of DWT- OFDM transmitter and receiver is shown in figure 3 and 4.Implementation of DWT requires only two filters, a low pass filter and a high pass filter, which makes the implementation simple in terms of complexity. It is clear that there is no cyclic prefix used in DWT-OFDM systems due to the overlapping properties of DWT, the most of the data is present in the main lobe, since sidelobes contain very low data hence the amount of interference is very low in DWT [13].

\section{A. Transmitter}

A random sequence of bits is generated. The number of bits is determined according to the number of sub-carriers required, number of bits per symbol and number of OFDM symbols used in the simulation. For this simulation the number of subcarriers used is 64, 6 bits per symbol for 64-DAPM and 1200 OFDM Symbols, by a simple calculation the number of generated bits were 460000 , which yielded a total amount of about 76000 samples for all the OFDM symbols which is a very huge data to test the performance of the system. 


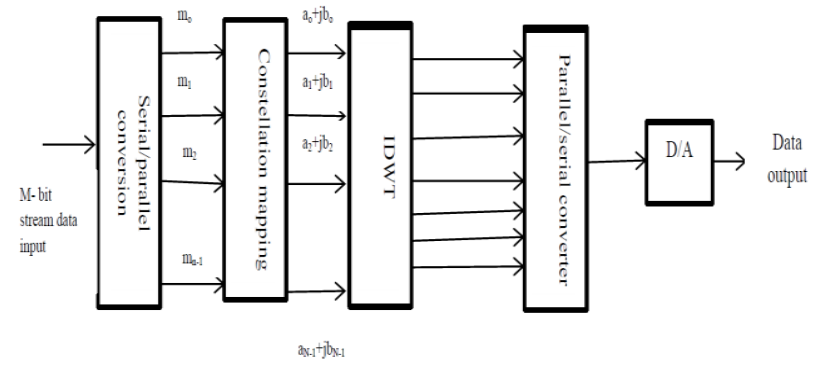

Fig. 3 Block diagram of DWT- OFDM transmitter

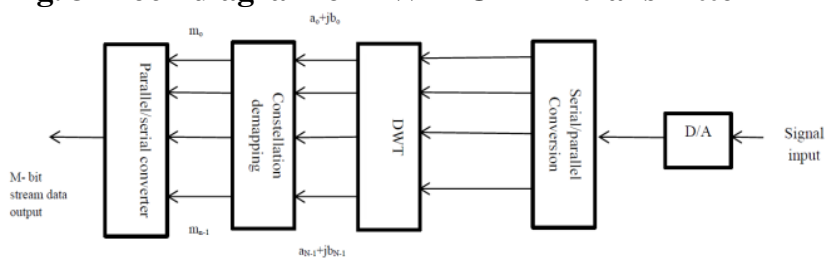

Fig. 4 Block diagram of DWT- OFDM Receiver

Before the bits are modulated they were divided into groups of six and put into a matrix form. The matrix consists of $\mathrm{N}$ rows which are the number of sub-carriers the signal is converted from serial to parallel, each column in the matrix represents an OFDM symbol. The first four bits of every symbol were modulated using ordinary 16-DAPM and the last two bits were used for the amplitude demodulation according to table I. The value of the amplitude parameter found in table 1 was set to 1.4 as it was found [14] that 1.4 is the optimum value for perfect demodulation. Figure 5 shows the constellation diagram simulated for a sequence of randomly generated bits.

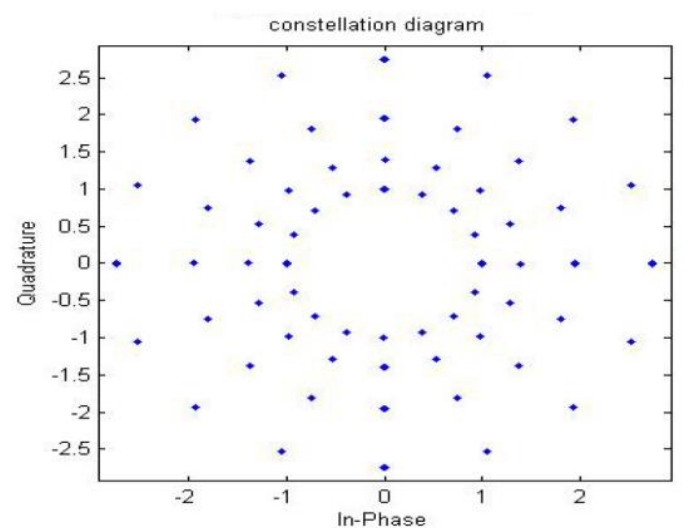

Fig. 5 Simulated 64-DAPM constellation diagram

Table-I: Amplitude values for 64- DAPM

\begin{tabular}{|c|c|c|c|}
\hline \multicolumn{5}{|c|}{ Last two Amplitude bits } \\
\hline 00 & 01 & 10 & 11 \\
\hline 1 & $\mathrm{a}$ & $\mathrm{a}^{2}$ & $\mathrm{a}^{3}$ \\
\hline $\mathrm{a}$ & $\mathrm{a}^{2}$ & $\mathrm{a}^{3}$ & 1 \\
\hline $\mathrm{a}^{2}$ & $\mathrm{a}^{3}$ & 1 & $\mathrm{a}$ \\
\hline $\mathrm{a}^{3}$ & 1 & $\mathrm{a}$ & $\mathrm{a}^{2}$ \\
\hline
\end{tabular}

The performance of a DWT based OFDM system adopting 64-DAPM as the modulation scheme is evaluated. To correctly evaluate the performance of the DWT based system, A DFT based system and a DCT based system both adopting a 64-DAPM, QAM and PAM scheme were also implemented to compare the results of the DWT based

$\mathrm{v}(\mathrm{n})=\mathrm{y}(\mathrm{n}) * \mathrm{z}(\mathrm{n})+\mathrm{w}(\mathrm{n})$ system. The IDWT block is the inverse process of the DWT block. To implement the IDWT only two filters are required a low-pass and a high-pass filter. Figure 6 shows the IDWT implementation, where $D_{K}$ is the $K_{t h}$ OFDM symbol after being modulated and $O_{k}$ is a vector of zeros having the same number of elements as the OFDM symbol. It is clear that the modulated signal $D_{k}$ is upsampled and then filtered using the low-pass filter $\mathrm{L}[\mathrm{n}]$, as for $O_{k}$ it is also upsampled and filtered but using the high-pass filter $\mathrm{H}[\mathrm{n}]$. Then the filter outputs are added together. The IDWT process is iterative which means that the same procedure can be repeated as many times as required. Both filters coefficients are determined according to which wavelet family is used, in this work the haar wavelets are used.

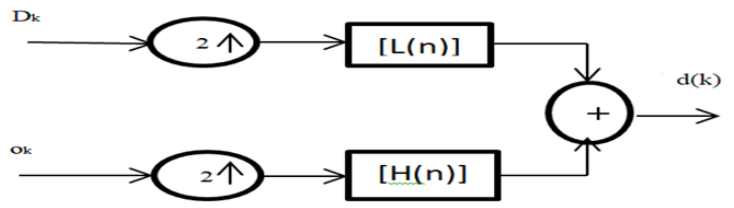

Fig.6 IDWT filter implementation.

\section{B. Channel}

In wireless communication the channel is always unpredictable. The signal traveling from the transmitter to the receiver suffers from very harsh conditions, such as fading, noise and Doppler spread. The first channel impairment is the noise in the channel which causes the signal to attenuate. Noise in the channel is modelled as an AWGN. AWGN is a result of several independent noise sources and is added to the signal. The main channel impairment is fading, fading occurs due to multipath reflections. The transmitted signal experience several reflections from objects between the transmitter and the receiver, resulting in simultaneous reception of the signal from different paths. The fading in this work is modelled as Rayleigh fading, where the magnitude of the signals arriving at the receiver have a Rayleigh distribution. In Rayleigh fading there is no line of sight component and the receiver only depends on the reflected components. Rayleigh fading can be either flat or frequency selective, frequency selective fading occurs when the signal's coherence bandwidth of the channel is smaller than the signals bandwidth and flat fading occurs when the coherence bandwidth of the channel is greater than the signals bandwidth. When the transmitter or the receiver are in motion this causes a Doppler effect which also affects the transmitted signal [14]. The Doppler Effect is relative to the speed of motion, the higher the speed is higher the effect and vice versa. The received signal after passing through the channel can be represented as follows:

Where $\mathrm{v}(\mathrm{n})$ is the received signal, $\mathrm{y}(\mathrm{n})$ is the transmitted signal, $\mathrm{z}(\mathrm{n})$ is the channel response and $\mathrm{w}(\mathrm{n})$ represents the added noise.

\section{Receiver}

The DWT filter implementation is shown in figure 7 and requires the same two filters which are used for IDWT filter implementation. 
The signal which received is filtered two times with the low pass filter once and with the high pass filter once and this process splits the signal into two equal halves for the analysis of low and high frequency components, then the output of the two filters is then down sampled. The output of the high-pass filter contains a small amount of information and is ignored. This process is also iterative. The number of iterations this time depends on the number of iterations used in the IDWT block. The output of the low-pass filter is always used as the input signal for the next iteration and finally the output of the low-pass filter after the final iteration is passed to the next block.

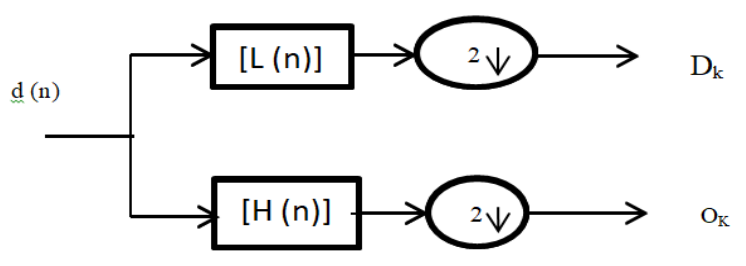

Fig. 7 DWT filter implementation

The demodulation is performed by 64-DAPM. The first four bits are determined by the phase of the received symbols by performing an ordinary 16-DAPM demodulation [14]. The last two bits are determined by the amplitude of the received symbols according to certain thresholds.

\section{RESULTS AND DISCUSSION}

Two performance indices PAPR and BER are considered for the proposed system. In the channel considered the Noise is modelled as AWGN with Rayleigh fading which is of frequency selective and flat fading channels.

\section{A. PAPR PERFORMANCE}

The comparison of PAPR performance of proposed OFDM system by using DAPM, QAM and PAM modulation techniques and different transforms such as DWT, DFT and DCT is done by CCDF plots. The CCDF is plotted for the combination of DWT-DAPM, DWT- QAM, DWT- PAM, DFT-DAPM, DFT-QAM, DFT- PAM, DCT-DAPM, DCTQAM, DCT- PAM.PAPR of DWT - DAPM combination is 4.497 at $10-{ }^{3}$ CCDF, DWT-QAM has the PAPR of 4.684 at $10-^{3}$ CCDF, DWT- PAM has the PAPR of 6.211 at $10^{-3}$ CCDF and all other combinations using different modulation techniques such as DAPM, QAM, PAM and other transforms such as DFT and DCT are as shown in figure 8 and all the results are tabulated in table II. From these results by using DWT and DAPM techniques PAPR is reduced from other techniques. Figure 9 shows PAPR for different modulation techniques and shows that DAPM modulation is better modulation technique and gives better reduction of PAPR. Figure 10 shows PAPR for different transforms and from this graph, DWT is the better method to reduce PAPR and the PAPR value is less compared to other transforms such as DFT and DCT.

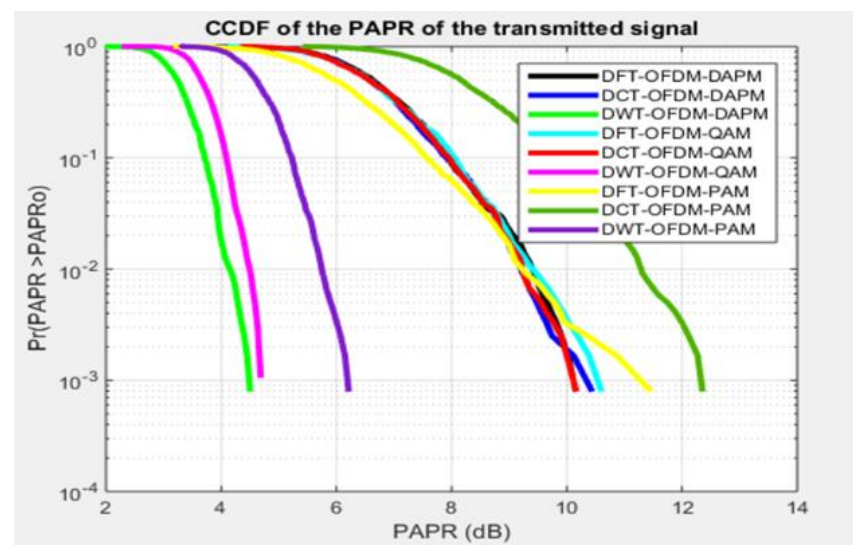

Fig. 8 PAPR and CCDF performances by using different transforms and modulation techniques

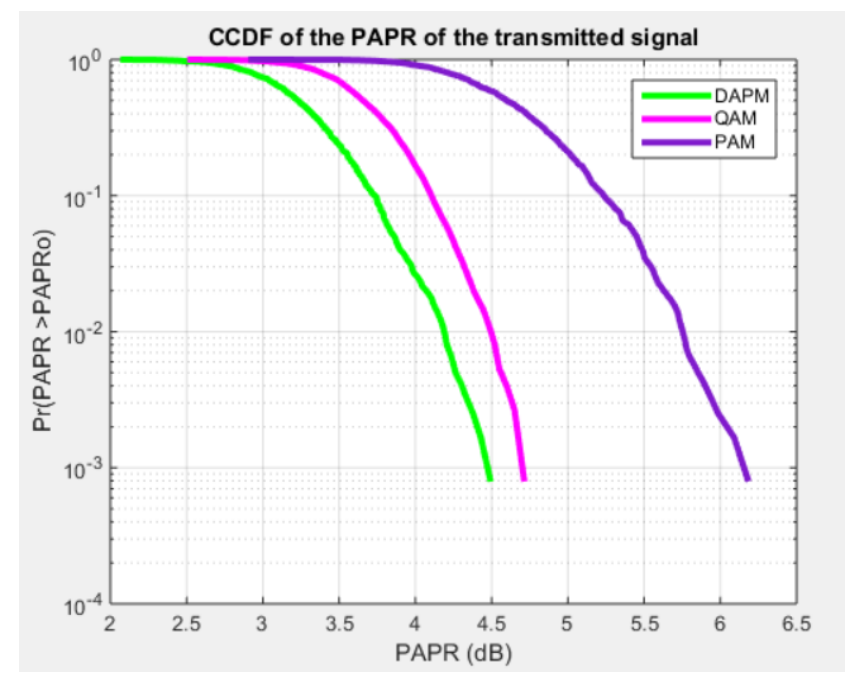

Fig. 9 PAPR and CCDF performances by using different modulation techniques using DWT

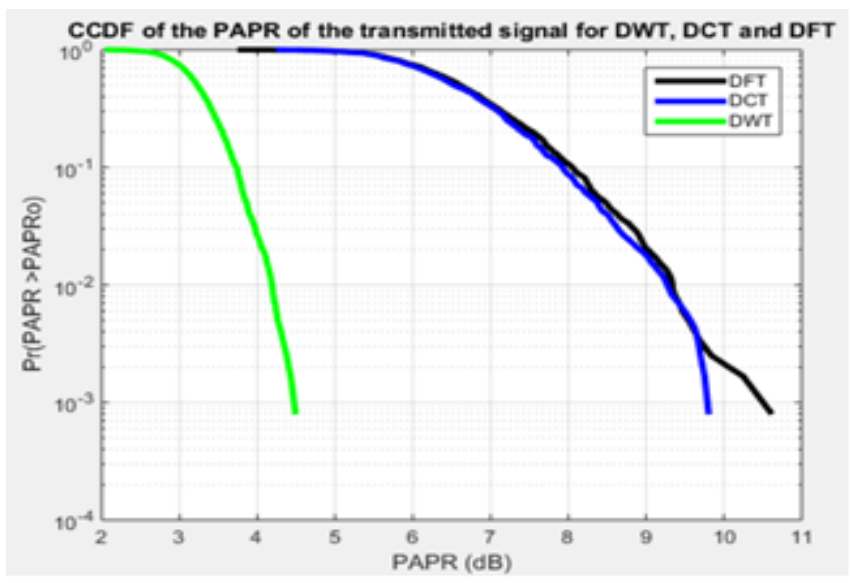

Fig. 10 PAPR and CCDF performances by using different transforms

\section{B. BER PERFPRMANCE}

The performance of the proposed system is compared by using the different modulation techniques and transforms. Here AWGN is used as a noise model and different fading channels such as flat fading and frequency selective fading is used and as shown in figure 11, 12, 13,14 and 15 these results are compared and tabulated in table III,IV and V. 
From these results DWT with QAM has a BER of 0.0008898 with SNR of $20 \mathrm{~dB}$ which is the least BER when the AWGN channel is considered, but by using frequency fading and flat fading channel with DAPM modulation technique.BER is least in the DAPM modulation technique. Table VI provides the comparative results with the proposed system and existing work and shows that our proposed system has better reduced PAPR and less BER than the existing works. Table VII shows the parameters used for simulation.

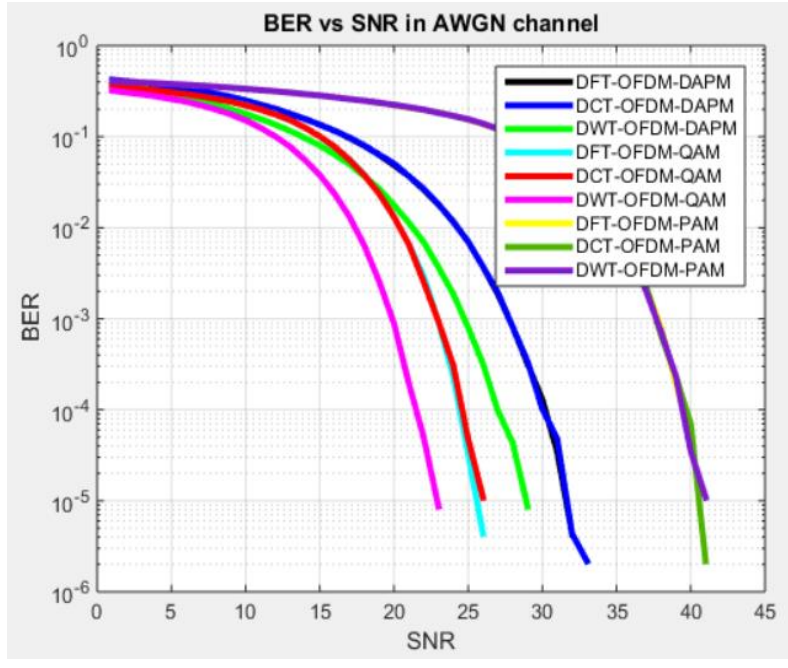

Fig. 11 BER performances by using different modulation techniques and transforms

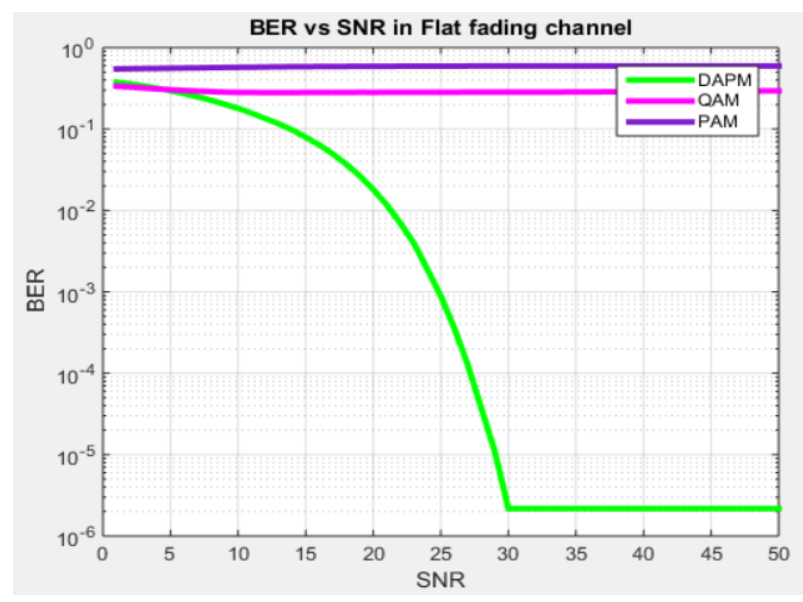

Fig. 14 BER performances of different modulation techniques in the flat fading channel using DWT

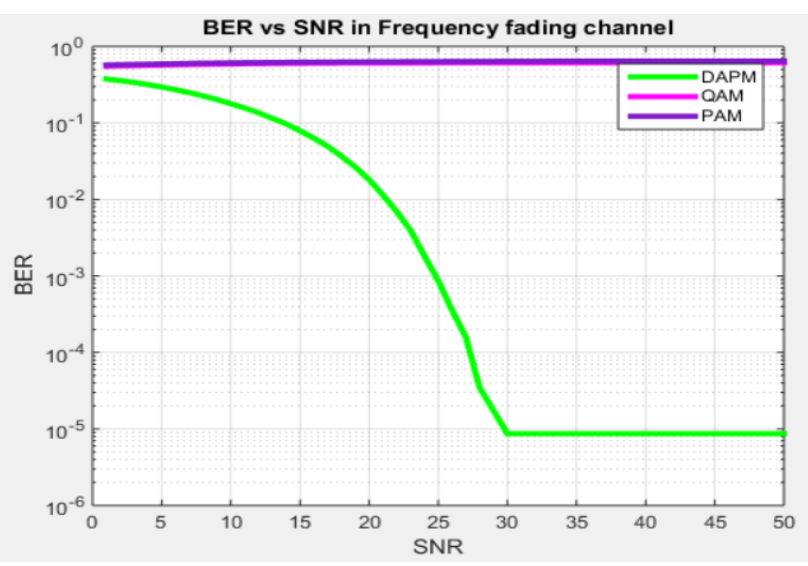

Table- II: PAPR and CCDF performances by using different modulation techniques and Transforms at 10-3 CCDF

\begin{tabular}{|l|c|}
\hline $\begin{array}{c}\text { Transform- Modulation } \\
\text { Technique }\end{array}$ & PAPR (dB) \\
\hline DWT-DAPM & 4.497 \\
\hline DWT-QAM & 4.684 \\
\hline DWT-PAM & 6.211 \\
\hline DFT-DAPM & 10.45 \\
\hline DFT-QAM & 10.59 \\
\hline DFT-PAM & 11.43 \\
\hline DCT-DAPM & 10.42 \\
\hline DCT-QAM & 10.15 \\
\hline DCT-PAM & 12.35 \\
\hline
\end{tabular}

Table- III: Comparisons of BER by using different transforms and modulation techniques using AWGN channel at $20 \mathrm{~dB}$ SNR

\begin{tabular}{|l|l|}
\hline $\begin{array}{c}\text { Transform- Modulation } \\
\text { Technique }\end{array}$ & BER \\
\hline DWT-DAPM & 0.01765 \\
\hline DWT-QAM & 0.00088 \\
\hline DWT-PAM & 0.2232 \\
\hline DFT-DAPM & 0.05062 \\
\hline DFT-QAM & 0.01313 \\
\hline DFT-PAM & 0.2232 \\
\hline DCT-DAPM & 0.05062 \\
\hline DCT-QAM & 0.01313 \\
\hline DCT-PAM & 0.2232 \\
\hline
\end{tabular}

Table-IV: Comparisons of BER by using different modulation techniques with Flat Fading Channel using DWT at 20dB SNR.

\begin{tabular}{|l|l|l|l|}
\hline $\begin{array}{l}\text { Modulation } \\
\text { Technique }\end{array}$ & DAPM & QAM & PAM \\
\hline BER & 0.01806 & 0.2816 & 0.5896 \\
\hline
\end{tabular}

Table- V: Comparisons of BER by using different modulation techniques with Frequency selective Fading Channel using DWT at 20dB SNR.

\begin{tabular}{|l|l|l|l|}
\hline $\begin{array}{l}\text { Modulation } \\
\text { Technique }\end{array}$ & DAPM & QAM & PAM \\
\hline BER & 0.01816 & 0.6278 & 0.6278 \\
\hline
\end{tabular}

Table- VI: Comparative results of the proposed system with the existing works.

\begin{tabular}{|c|c|c|c|}
\hline $\begin{array}{c}\text { Performance } \\
\text { indices }\end{array}$ & PAPR (dB) & $\begin{array}{c}\text { BER } \\
\text { (dB) }\end{array}$ & $\begin{array}{c}\text { Modulation } \\
\text { Technique }\end{array}$ \\
\hline $\begin{array}{c}\text { Proposed } \\
\text { System }\end{array}$ & $\begin{array}{c}4.497 \text { at } 10^{-3} \\
\text { CCDF }\end{array}$ & $\begin{array}{c}0.01816 \\
\text { at } \\
20 \mathrm{~dB} \\
\text { SNR }\end{array}$ & DAPM \\
& & ---------- & QAM \\
\hline Reference 3 & $\begin{array}{c}\text { 8.21 at } 10^{-3} \\
\text { CCDF }\end{array}$ & & \\
\hline
\end{tabular}




\begin{tabular}{|c|c|c|c|}
\hline Reference 4 & -------------- & $10^{-3} \mathrm{~dB}$ at & QAM \\
& & $12 \mathrm{~dB}$ & \\
& & SNR & \\
\end{tabular}

Table -VII: Considered parameters while simulation

\begin{tabular}{|c|c|c|}
\hline $\begin{array}{c}\text { Sl } \\
\text { No. }\end{array}$ & Parameters & $\begin{array}{c}\text { Proposed } \\
\text { System }\end{array}$ \\
\hline 1 & Number of Subcarriers & 64 \\
\hline 2 & $\begin{array}{c}\text { Number of bits per } \\
\text { symbol }\end{array}$ & 6 \\
\hline 3 & $\begin{array}{c}\text { Number of OFDM } \\
\text { symbols }\end{array}$ & 1200 \\
\hline 4 & Number of bits & $4,60,800$ \\
\hline 5 & Cyclic Prefix use & NO \\
\hline 6 & Modulation use & DAPM \\
\hline
\end{tabular}

\section{CONCLUSION}

In this paper, the proposed DWT based OFDM system using DAPM modulation technique along with DFT and DCT based OFDM systems with modulation techniques QAM, PAM are considered and compared. By using the DWT, PAPR is cut by $5.653 \mathrm{~dB}$ and BER vs SNR is evaluated in AWGN channel, frequency selective fading and flat fading channel. Thus, the proposed system performance is better compared to DFT and DCT. The proposed OFDM is useful for digital television and $4 \mathrm{G}$ mobile communication.

\section{REFERENCES}

1. Gerhard Wunder, Robert F.H. Fischer, Holger boche, Simon Litsyn, and Jong-Seon No, "The PAPR problem in OFDM transmission" ,IEEE signal processing magazine, November 2013, pp.130-144.

2. Dae-Woon Lim, Jong-Seon No, Chi-Woo Lim, and Habong Chung, "A New SLM OFDM Scheme With Low Complexity for PAPR Reduction", IEEE Signal processing letters, Vol. 12, no. 2, February 2005,pp. 93-96.

3. Alok Joshi, Apoorv Manas, Samarth Garg and Rahul Wason, "PAPR Reduction Comparison in FFT-Based OFDM Versus DWT-Based OFDM",Advances in Signal Processing and Communication , 2019, pp. 107-115.

4. A.Vani ,Pranali Shanker Bargade, "Improvement of BER for Mimo-Ofdm System Using Wavelet Transform", International Journal of Innovative Technology and Exploring Engineering (IJITEE)", Volume-8 Issue-8 June, 2019 pp.204-207.

5. Swati Patel, Vipul Awasthi, Pankaj Sahu," PAPR Reduction in OFDM system using m2m DWT scheme", Journal of Emerging Technologies and Innovative Research (JETIR)", Volume 4, Issue 03, March 2017, pp.134-138.

6. ÖĞR.GÖR. MURAT TÖREN, "PAPR Reduction with Wavelet Transform and Different PAPR Reduction Tecniques in MIMO-OFDM Systems", Journal of Engineering and Architecture, Vol. 1 No. 2, December 2013, pp. 10-21.

7. Ali Jasim Ghaffoori," PAPR Reduction in OFDM system using adaptive hybrid technique", IOP Conference Series: Materials Science and Engineering,, 2019, pp.052021.

8. Kelvin Anoh, Augustine Ikpehai, Khaled Rabie, Bamidele Adebisi and Wasiu Popoola, "PAPR Reduction of Wavelet-OFDM Systems Using Pilot Symbols",IEEE International Symposium on Power Line Communications and its Applications (ISPLC), 2018, pp.1-6.

9. Murat Tören \& Cebrail Çiflikli, "Peak-to-Average-Power-Ratio (PAPR) Reduction Methods with Wavelet Transform in MIMO-OFDM", IETE journal of research, 2017, pp. 1-7.
10. Busra Ceniklio $\div$ lu*, AlOzent, and Ibrahim Develi, "ZT DWT-S OWDM: A New Waveform for PAPR Reduction", $41^{\text {st }}$ International Conference on Telecommunications and Signal processing (TSP),2018, pp. 1-4.

11. Manuvinakurke Narasimhasastry suma, Somenahalli venkatarangachar Narasimhan, Buddi Kanmani, “ Orthogonal frequency division multiplexing peak to average power ratio reduction by best tree selection using coded discrete cosine harmonic wavelet packet transform", IET communications, vol. 8, Iss.11,2014, pp. 1875-1882.

12. Abbas Ali Sharifi, "A new post-coding approach for PAPR reduction in DC-biased optical OFDM systems", optoelectronics letters, vol.15, No.4, 1 July, 2019, pp. 302-305.

13. Ye,sim HEK IM TANÇ, Aydm Akan, "DWT Based OFDM with DAPSK Modulation for Power Line Communication Systems", $25^{\text {th }}$ Signal processing and communications Applications Conference ( SIU), , 2017, pp.01-07.

14. H. Y. S. Xu and H. Wang, "An application of dapsk in hf communications," IEEE COMMUNICATIONS LETTERS, vol. 9, July. 2005, pp. 613-615.

\section{AUTHORS PROFILE}

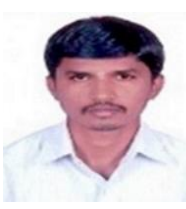

Puneeth Kumar D. N., received his Bachelor's degree in Telecommunication Engineering in 2009 from Visvesvaraya Technological University-Belagavi, India, M.Tech in Digital Electronics and Communication Systems from Visvesvaraya Technological University- Belagavi, India in 2012. He is currently working as Assistant Professor in the Department of Telecommunication Engineering at Sri Siddhartha Institute of Technology, Tumkur, India and also pursuing his PhD from Visvesvaraya Technological University-Belagavi, India. He published 1 paper in International Journal, 4 Papers in International Conferences, and 2 in National Conferences. His teaching and research interests are in the field of wireless communication, signal processing

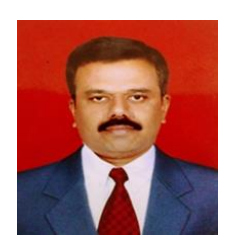

M. N. Eshwarappa, is presently working as Professor, Dept. of ECE, SSIT-Tumkur,India. He completed his B.E from Mysore University, India in the year 1991, M.Tech from Mangalore University, KREC-Surathkal, in the year 1999 and $\mathrm{PhD}$ from Visvesvaraya Technological University-Belagavi, India in the year 2013. He has more than 26 years of Teaching and 6 years of research experience. His research interests are Communication, Biometrics, Medical Imaging and Signal Processing. He has more than 08 International Journal papers and 30 National/International Conference publications to his credit. 